\title{
Carboplatin-induced Renal Salt Wasting Syndrome in Paediatric Patients with Intracranial Germ Cell Tumours and Concomitant Diabetes Insipidus
}

\author{
Tania Wan ${ }^{1}$, Joanna Tung ${ }^{2}$, Samantha Lee ${ }^{2}$, Alison LT Ma ${ }^{3}$, Anthony Pak Yin Liu ${ }^{4}$, \\ Dennis $\mathrm{Ku}^{5}$, Ping-wa Yau ${ }^{2}$, and Matthew Shing ${ }^{2}$ \\ ${ }^{1}$ Queen Mary Hospital \\ ${ }^{2}$ Hong Kong Children's Hospital \\ ${ }^{3}$ Princess Margaret Hospital \\ ${ }^{4}$ The University of Hong Kong \\ ${ }^{5}$ Hospital for Sick Children
}

December 21, 2021

\begin{abstract}
We report the first case series of 14 children with intracranial germ cell tumour (iGCT) and concomitant central diabetes insipidus (DI), who developed hyponatremia secondary to renal salt wasting syndrome (RSWS) following the administration of carboplatin. Clinicians prescribing platinum-based chemotherapy for this group of patients should be alert to the risk of RSWS. Regular monitoring should be performed as hyponatraemia can be asymptomatic until it is severe.
\end{abstract}

Running title: Renal Salt Wasting Syndrome in Patients with Germ Cell Tumours

Carboplatin-induced Renal Salt Wasting Syndrome in Paediatric Patients with Intracranial Germ Cell Tumours and Concomitant Diabetes Insipidus

Tania Wan, Joanna Yuet-ling Tung, Alison Ma, Samantha Lai-Ka Lee, Anthony Pak-yin Liu, Dennis Tak-loi $\mathrm{Ku}$, Ping-wa Yau and Matthew Ming-kong Shing

Department of Paediatrics and Adolescent Medicine, Hong Kong Children's Hospital, Hong Kong

Correspondence:

Matthew Ming-kong Shing

Department of Paediatrics and Adolescent Medicine, Hong Kong Children's Hospital, 1 Shing Cheong Road, Kowloon, Hong Kong.

Phone: (852)-3513 3513. Fax: (852)-3512 7596.

Email: smk423@ha.org.hk

Word count (abstract): 67

Word count (main text): 1200

Abbreviation key: 


\begin{tabular}{ll}
\hline ADH & antidiuretic hormone \\
\hline AI & adrenal insufficiency \\
DI & diabetes insipidus \\
iGCT & intracranial germ cell tumour \\
RSWS & Renal salt wasting syndrome \\
NGGCT & non-germinomatous germ cell tumour \\
\hline
\end{tabular}

\begin{abstract}
We report the first case series of 14 children with intracranial germ cell tumour (iGCT) and concomitant central diabetes insipidus (DI), who developed hyponatremia secondary to renal salt wasting syndrome (RSWS) following the administration of carboplatin. Clinicians prescribing platinum-based chemotherapy for this group of patients should be alert to the risk of RSWS. Regular monitoring should be performed as hyponatraemia can be asymptomatic until it is severe.
\end{abstract}

\title{
Keywords
}

germ cell tumour; paediatrics; renal salt wasting syndrome; carboplatin

\section{Introduction}

Electrolyte disturbances are common in oncology patients, as many chemotherapy agents are nephrotoxic. ${ }^{1}$ Platinum-based chemotherapy is well known to be associated with electrolyte imbalances. ${ }^{2,3}$ One of its side effects is renal salt wasting syndrome (RSWS), which is characterised by hyponatraemia, in conjunction with polyuria and hypovolaemia. ${ }^{4-7}$

Patients with iGCTs are at risk of electrolyte disturbances, even before the administration of nephrotoxic chemotherapy. This is because iGCTs often arise in the suprasellar region, thereby potentially compressing on the pituitary gland. ${ }^{8}$ This results in pituitary dysfunction, such as central DI or central adrenal insufficiency (AI), which can in turn cause electrolyte disturbances. ${ }^{9}$

Platinum-based chemotherapy agents are highly active against a variety of solid tumours and are the main backbone of treatment for iGCTs. ${ }^{10}$ We describe the first case series of paediatric patients with central DI secondary to iGCT, all of whom developed concomitant RSWS following the administration of carboplatin. The combination of DI and RSWS and their respective effects on serum sodium levels, urine output and fluid balance made the diagnosis elusive and the management of these patients challenging.

Methods We performed a retrospective review of all paediatric patients (age $=/<19$ years at diagnosis), with newly diagnosed iGCTs and central DI managed at Hong Kong Children's Hospital from July 2019 until January 2021. Patients with the diagnosis of iGCT and DI were identified using the Clinical Data Analysis and Reporting System. Using electronic patient records, we reviewed the blood results, medications, and clinical details of this cohort. Patients were treated according to the Children's Oncology Group ACNS1123 study; induction chemotherapy consisted of 4 cycles of carboplatin $(600 \mathrm{mg} / \mathrm{m} 2 /$ day on Day 1$)$ and etoposide (150mg/m2/day on Day 1-3) for patients with germinomas, and alternating cycles of carboplatin-etoposide, and ifosfamide-etoposide for total of 6 cycles in patients with non-germinomatous germ cell tumours (NGGCT).

Results A total of 14 patients were identified to have newly diagnosed iGCT from July 2019 to January 2021, 10 had germinomas and 4 had NGGCTs. Table 1 describes their clinical characteristics. In addition to central DI, all patients also had anterior pituitary hormonal deficiencies, including 13 who had central AI. Seven patients underwent tumour biopsy, 2 underwent tumour resection and 5 had no neurosurgical procedures performed prior to chemotherapy. All patients were administered carboplatin $600 \mathrm{mg} / \mathrm{m}^{2} /$ day for 1 day and etoposide $150 \mathrm{mg} / \mathrm{m} 2 /$ day for 3 days as their first induction cycle. One patient had adipsic DI 
and had a baseline serum sodium level of $160 \mathrm{mmol} / \mathrm{L}$; all others had normal baseline serum sodium levels prior to chemotherapy. All 14 patients were found to have hyponatraemia on routine bloods following the first cycle of carboplatin despite significant diuresis of more than $3 \mathrm{~L} / \mathrm{m} 2 /$ day and negative fluid balance. The nadir sodium level ranged from 122 to $134 \mathrm{mmol} / \mathrm{L}$ (mean $128 \mathrm{mmol} / \mathrm{L}$ ). Paired urine sodium levels at times of hyponatremia were noted to be inappropriately high, ranging from 40 to $>250 \mathrm{mmol} / \mathrm{L}$ (urine sample saved before commencement of sodium supplementation). In anticipation of chemotherapy-related emesis, all patients with AI were covered with stress-dose hydrocortisone at $30 \mathrm{mg} / \mathrm{m} 2 /$ day.

Ten patients required sodium supplementation (range: $1.6-11.8 \mathrm{mmol} / \mathrm{kg} /$ day), of which 8 patients required intravenous supplementation. In addition to hyponatraemia, all patients concurrently had at least one of following: hypokalaemia, hypocalcaemia or hypophosphataemia. Two patients developed hypotension and 3 required fludrocortisone treatment.

All 14 patients in this series received a total of 36 subsequent cycles of carboplatin following the first cycle of chemotherapy. Hyponatraemia with serum sodium ranging 118-134mmol/L (median 132mmol/L) was noted following 16 (44.4\%) of these cycles. Paired urinary sodium was again noted to be high during these episodes (97 to $>250 \mathrm{mmol} / \mathrm{L})$. All patients were noted to have normal long-term renal function.

DiscussionWe report the first case series of patients with iGCT and central DI, complicated with concomitant RSWS following the administration of carboplatin. Central DI is characterized by hypernatraemia and the passing of large amounts of dilute urine, due to a lack of antidiuretic hormone (ADH). ${ }^{11}$ The lack of $\mathrm{ADH}$ renders the kidneys unable to concentrate urine and consequently, patients with DI experience hypernatraemia, polyuria and hypovolaemia. The treatment of central DI is by the administration of desmopressin, a synthetic form of $\mathrm{ADH}$.

RSWS similarly presents with excessive diuresis, but is characterized by hyponatraemia, rather than hypernatraemia, as large amounts of electrolytes are lost in the urine due to tubular damage, leading to a high urinary sodium. ${ }^{4-6,12}$ RSWS is associated with the depletion of extracellular volume and in severe cases, hypotension, as large amounts of fluid are drawn out into the urine. It causes salt loss and is therefore often associated with other electrolyte disturbances such as hypokalaemia, hypophosphataemia and hypocalcaemia. ${ }^{3,13}$ The treatment of RSWS is with volume repletion and salt replacement; mineralocorticoids such as fludrocortisone may also be effective. ${ }^{14}$

Platinum-based chemotherapy is well-known for causing electrolyte balances including hyponatraemia, hypophosphataemia, hypocalcaemia and hypokalaemia. RSWS is a rare reported side effect; however, the mechanism is not well understood. ${ }^{4,6}$ Platinum-based chemotherapy causes renal damage via accumulation in the proximal and distal epithelial cells and the most likely site for damage in RSWS is the proximal nephron, which is responsible for the majority of sodium reabsorption. ${ }^{3,15}$

Carboplatin has previously been reported in a paediatric patient to cause recurrent RSWS, but there was no concomitant DI. ${ }^{4}$ In adults, cisplatin-induced renal salt wasting has been previously documented. ${ }^{5}$ Similar to our case series, concomitant central DI and RSWS was described in a paediatric patient following the administration of platinum-based chemotherapy. However, cisplatin, rather than carboplatin was the culprit. ${ }^{7}$ The observation of RSWS after carboplatin in children with iGCT and concurrent DI is not well reported in the literature, probably due to the low incidence of iGCT among non-Asians.

The majority of patients in our series have central adrenal insufficiency. As a routine practice in our centre, these patients are prescribed stress dose hydrocortisone to prevent relative adrenal insufficiency related to vomiting during chemotherapy. Therefore, renal salt wasting secondary to relative adrenal insufficiency can be excluded. All patients in our cohort developed salt wasting with hyponatraemia to some extent following the first administration of carboplatin. This occurred regardless of the type of iGCT, (i.e., germinoma or NGGCT), and regardless of any prior neurosurgical procedure.

All patients were noted to have at least one of the following: hypocalcaemia, hypophosphataemia and/or hypokalaemia, which are commonly observed in RSWS. Furthermore, urine $\beta 2$ microglobulin levels were 
also high in some of our patients, confirming renal tubular damage. Some patients required fludrocortisone for treatment, following which, the amount of sodium loss through the tubules significantly decreased, as evidenced by a drop in urinary sodium and stabilization of serum sodium levels. Of note, some patients experienced only mild hyponatraemia, which was detected on routine bloods. This indicates that RSWS may present in a milder form that it is likely under-recognised. Although RSWS did not always recur with each cycle of carboplatin, recurrence was common (44.4\%), and the ensuing hyponatraemia could still be severe.

The limitations of this case series are its retrospective nature and small sample size. Though review of clinical notes and laboratory results consistently showed that hyponatraemia in these patients occurred following the first cycle of carboplatin, not all had extensive workup performed, such as pro-BNP, urinary amino acids or $\beta 2$ microglobulin, particularly if the hyponatraemia was mild and self-resolved without further intervention. Nevertheless, this case series illustrates that this group is vulnerable to electrolyte disturbances following administration of carboplatin and underscores the need for closer monitoring during treatment.

Conflict of interest: The authors have no conflicts of interest to declare.

References

1. Ruggiero A, Ferrara P, Attinà G, Rizzo D, Riccardi R. Renal toxicity and chemotherapy in children with cancer. Br J Clin Pharmacol . 2017;83(12):2605-2614. doi:10.1111/bcp.13388

2. English MW, Skinner R, Pearson AD, Price L, Wyllie R, Craft AW. Dose-related nephrotoxicity of carboplatin in children. Br J Cancer . 1999;81(2):336-341. doi:10.1038/sj.bjc.6690697

3. Oronsky B, Caroen S, Oronsky A, et al. Electrolyte disorders with platinum-based chemotherapy: mechanisms, manifestations and management.Cancer Chemother Pharmacol . 2017;80(5):895-907. doi:10.1007/s00280-017-3392-8

4. Tscherning C, Rubie H, Chancholle A, et al. Recurrent renal salt wasting in a child treated with carboplatin and etoposide.Cancer . 1994;73(6):1761-1763. doi:10.1002/1097-0142(19940315)73:6<1761::aidcncr2820730635>3.0.co;2-\#

5. Cao L, Joshi P, Sumoza D. Renal salt-wasting syndrome in a patient with cisplatin-induced hyponatremia: case report. Am J Clin Oncol . 2002;25(4):344-346. doi:10.1097/00000421-200208000-00005

6. Hamdi T, Latta S, Jallad B, Kheir F, Alhosaini MN, Patel A. Cisplatin-induced renal salt wasting syndrome. South Med J . 2010;103(8):793-799. doi:10.1097/SMJ.0b013e3181e63682

7. Cortina G, Hansford JR, Duke T. Central Diabetes Insipidus and Cisplatin-Induced Renal Salt Wasting Syndrome: A Challenging Combination. Pediatr Blood Cancer . 2016;63(5):925-927. doi:10.1002/pbc.25910

8. Jennings MT, Gelman R, Hochberg F. Intracranial germ-cell tumors: natural history and pathogenesis. $J$ Neurosurg . 1985;63(2):155-167. doi:10.3171/jns.1985.63.2.0155

9. García García E, Gómez Gila AL, Merchante E, et al. Endocrine manifestations of central nervous system germ cell tumors in children.Endocrinol Diabetes Nutr . 2020;67(8):540-544. doi:10.1016/j.endinu.2019.11.012

10. Frazier AL, Stoneham S, Rodriguez-Galindo C, et al. Comparison of carboplatin versus cisplatin in the treatment of paediatric extracranial malignant germ cell tumours: A report of the Malignant Germ Cell International Consortium. Eur J Cancer Oxf Engl 1990 . 2018;98:30-37. doi:10.1016/j.ejca.2018.03.004

11. Maghnie M, Cosi G, Genovese E, et al. Central Diabetes Insipidus in Children and Young Adults. $N$ Engl J Med . 2000;343(14):998-1007. doi:10.1056/NEJM200010053431403

12. Inamori M, Inohara H, Horii A. Differential diagnosis of hyponatremia induced by cisplatin-containing chemotherapy: syndrome of inappropriate secretion of antidiuretic hormone (SIADH) or renal salt wasting syndrome (RSWS). Acta Oto-Laryngol Case Rep . 2016;1(1):33-35. doi:10.1080/23772484.2016.1198231 
13. Liamis G, Milionis H, Elisaf M. A review of drug-induced hyponatremia. Am J Kidney Dis Off J Natl Kidney Found . 2008;52(1):144-153. doi:10.1053/j.ajkd.2008.03.004

14. Ghosh S. Cisplatin: The first metal based anticancer drug.Bioorganic Chem . 2019;88:102925. doi:10.1016/j.bioorg.2019.102925

15. Hanigan MH, Devarajan P. Cisplatin nephrotoxicity: molecular mechanisms. Cancer Ther . 2003;1:47-61.

\section{Hosted file}

Summary table_final.docx available at https://authorea.com/users/452174/articles/ 550311-carboplatin-induced-renal-salt-wasting-syndrome-in-paediatric-patients-withintracranial-germ-cell-tumours-and-concomitant-diabetes-insipidus 